\title{
Investigations on the edge kinetic data in regimes with type-I and mitigated ELMs at ASDEX Upgrade
}

\author{
S K Rathgeber, L Barrera, G Birkenmeier, R Fischer, \\ W Suttrop and the ASDEX Upgrade Team \\ Max-Planck-Institut für Plasmaphysik, EURATOM Association, \\ Boltzmannstraße 2, 85748 Garching, Germany \\ E-mail: sylvia.rathgeber@ipp.mpg.de
}

\begin{abstract}
.
The behaviour of profiles and gradients of electron density, temperature and pressure at the edge of ASDEX Upgrade was studied in regimes with type-I and small Edge Localized Modes (ELMs) of discharges with and without applied Magnetic Perturbations (MPs). Estimation of the edge kinetic parameters was performed by means of integrated data analysis for joint reconstruction of electron density and temperature profiles via combination of data from different diagnostics. The MP fields for ELM mitigation were produced by 16 in-vessel coils allowing to execute this survey with large variations in poloidal spectrum and resonant component of the error field.

With several dedicated discharges the effect of MPs on the edge kinetic data and ELMs was determined in dependence of heating power, gas puff and MP-coil configuration. Small ELMs are dominant - with and without MPs — in regimes with reduced pedestal top electron temperatures and flattened edge electron pressure gradients compared to type-I ELM phases. Furthermore, application of MPs opens an additional small ELM regime in the high temperature range at reduced electron pressure gradient.
\end{abstract}

\section{Introduction}

Mitigation of type-I Edge Localized Modes (ELMs) is likely required for ITER operation in order to reduce the heat flux on the divertor plates and first wall components. In present day machines, application of non-axisymmetric Magnetic Perturbation (MP) fields at the plasma edge by external coils has been found to be a promising technique to achieve stable H-mode plasmas without ELMs or with small ELMs and appropriate confinement properties $[1,2,3,4]$. However, the underlying mechanism is not yet understood and detailed investigations on the effect of MPs on the plasma are necessary to assess the possibility of ELM mitigation with MPs at ITER.

Since ELMs are assumed to be driven by a large edge pressure gradient and the consequent large bootstrap current density at the plasma boundary, the subject of this work is to assess the relation between MP-coil configuration, occurring ELM type and kinetic edge data at ASDEX Upgrade. A valuable tool for this study is provided by 
Integrated Data Analysis (IDA) [5] of different diagnostics to simultaneously evaluate electron density and temperature and, therefore, also the pressure in the framework of Bayesian probability theory. Recent improvements in modelling the Electron Cyclotron Emission (ECE) data within IDA [6] enable the reconstruction of accurate edge electron temperature profiles and gradients.

Based on this reliable method, a thorough investigation on the edge kinetic parameters in regimes with type-I and mitigated ELMs in the presence of different MP-coil configurations has been carried out. Generally, with and without MPs, the occurrence of small ELMs is related to higher densities and lower temperatures at the pedestal top and reduced edge pressure gradients compared to type-I ELMs. Additionally, in the presence of MPs small ELMs can also exist in the high temperature range at slightly lower edge pressure gradients than type-I ELMs.

The article is organized as follows: The estimation of the edge kinetic data via IDA is briefly introduced in section 2 and the MP-coil setup is sketched in section 3. The three following sections are restricted to three dedicated discharges with different MP-coil configurations whose design and overall behaviour (section 4), edge kinetic profiles (section 5) and edge pressure gradient evolution (section 6) are described in detail. These findings are complemented by further data to point out how the edge kinetic data generally are related to the occurring ELM type (section 7). The article ends with a discussion regarding the results in the light of two different hypotheses for ELM mitigation via MPs. Parts of this work have already been published in [7] and more details can be found in [8].

\section{Integrated data analysis}

The edge kinetic profiles were estimated by means of IDA which combined the data of different complementary diagnostics for joint analysis of electron density and temperature in the framework of Bayesian probability theory [9]. As in standard analysis, we thereby applied the LIthium Beam emission spectroscopy (LIB) and interferometry diagnostic for the density and ECE data for the temperature reconstruction [5].

Unlike previous straight forward ECE analysis [10] of identifying the electron temperature with the radiation temperature at the cold resonance position - where the measured frequency is equal to the second harmonic of the electron cyclotron frequency - we made use of the newly developed Electron Cyclotron Forward Modelling (ECFM) which is described in detail in [6]. With this method we achieved accurate electron temperature profiles and gradients also in the optically thin and steep edge region by calculating the broadened emission and absorption profiles and solving the radiation transport equation. One important feature of the ECFM method applied throughout all the analyses presented in this study was the automatic alignment of density and temperature profiles relative to each other by applying the 'separatrix criterion' [6]. The latter adapted the position of maximum curvature in density to the position of 

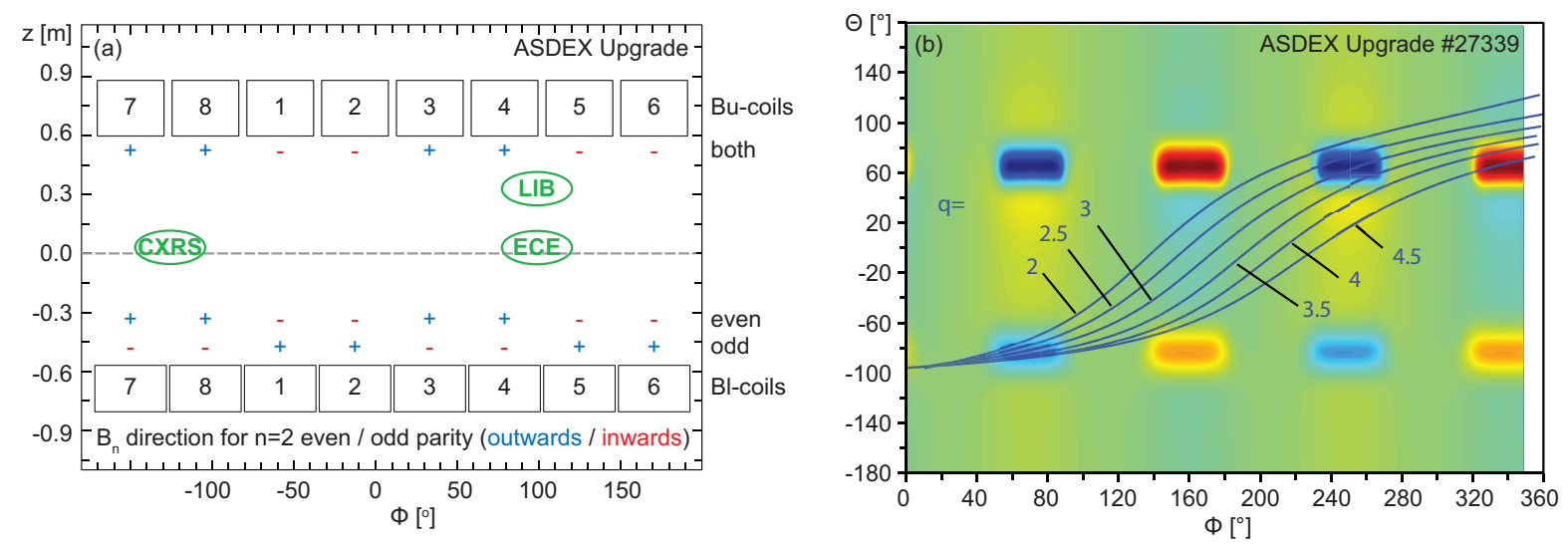

Figure 1. (a) Setup of the MP coils at ASDEX Upgrade in the $z-\Phi$ plane with the indicated current configuration for $n=2$ odd and even. (b) Alignment of the MP field with field lines at different safety factors [13].

$T_{\mathrm{e}}=100 \mathrm{eV}-$ a reasonable estimate for the upstream temperature predicted by models of the parallel heat conduction to the divertor [11]. This relation between the electron temperature and density profiles has been proposed in [12] and confirmed by sensitivity studies and comparison with Thomson Scattering measurements presented in [6].

To assess the profile uncertainties we applied the Markov Chain Monte Carlo (MCMC) method [9] which allowed to sample the complete posterior probability density function whose width delivered the uncertainties. In contrast to the usual $\chi^{2}$-binning method only providing the error bars of the profiles [9], MCMC calculations also deliver the uncertainties of the gradients.

\section{Magnetic perturbation coil setup and configuration}

The ASDEX Upgrade tokamak has recently been equipped with 16 MP coils for ELM mitigation [4]. They have been installed at eight equally distributed toroidal positions above and below the outer midplane. The left panel of figure 1 shows the two rows of coils in the $z-\Phi$ plane. The signs for the indicated coil currents corresponded to an error field with toroidal mode number $n=2$ with even or odd parity (same or opposite coil current in upper and lower coils). Positive/negative values of the coil current caused an outward/inward directed radial field component.

With different coil current polarity within each row it was possible to create nonaxisymmetric perturbation fields with toroidal mode numbers $n=1,2$ and 4 , while the phase between upper and lower coils determined the resonant field component whose ratio of poloidal $m$ and toroidal $n$ mode numbers matched the safety factor: $q=m / n$ (cf. figure 1(b)). Due to the box-like shaping of the coils which always created broad poloidal spectra [14] and the shaped diverted plasmas it was not possible to achieve fully resonant MPs. However, the amplitude of the resonant component was varied by an order of magnitude.

Also indicated on the left-hand side of figure 1 are the positions of the LIB and ECE 

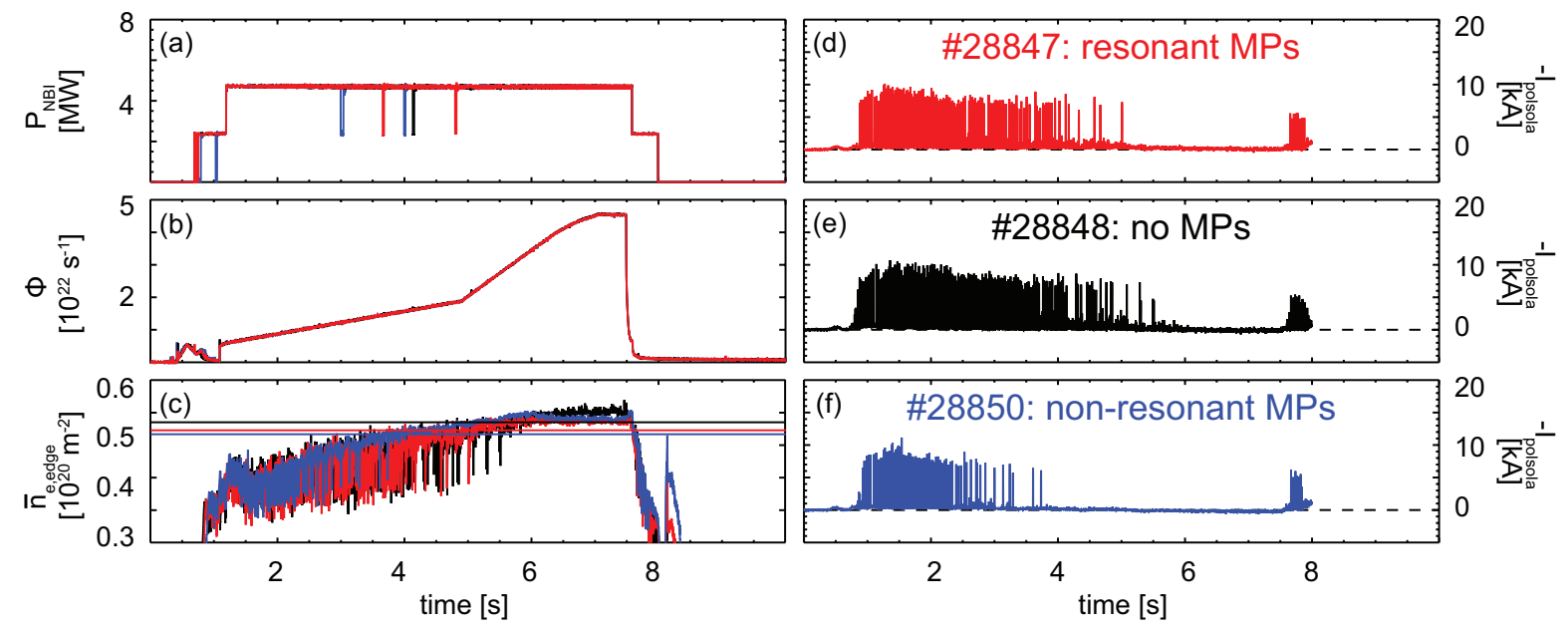

Figure 2. Time traces of (a) the NBI heating power, (b) the deuterium puff, (c) the line-averaged edge density and (d,e,f) the divertor current signals of discharges \#28847 with resonant MPs (red), \#28848 without MPs (black) and \#28850 with non-resonant MPs (blue).

diagnostics where the field line perturbation was caused by coils 5 and 6 . Since in all the presented discharges both of them possessed a positive coil current causing an outward bending of the field lines, the electron density the temperature profiles appeared on flux surfaces too far outside [15]. For the ECE reconstruction this misalignment was weaker, since the ECE diagnostics was located vertically further apart from the MP-coils. This justified an inward shift of the LIB data relative to the ECE data - as it was also suggested by the separatrix criterion (cf. section 5).

\section{Discharge description}

To investigate the effect of MPs on the edge electron density, temperature and pressure profiles, three identical discharges with different application of MPs were performed - one with resonant $n=2$ perturbation (\#28847), one with non-resonant $n=2$ perturbation (\#28850) and the reference discharge without MPs (\#28848). (The colour coding of data with resonant MPs indicated in shades of red, data with non-resonant MPs in shades of blue and data without MPs in shades of grey is used throughout the whole sections 4 to 7 .) In all three discharges the same NBI heating power of $P_{\mathrm{NBI}}=4.9 \mathrm{MW}$ (figure $2(\mathrm{a})$ ), a plasma current of $I_{\mathrm{P}}=800 \mathrm{kA}$ and a toroidal magnetic field of $B_{\mathrm{T}}=-2.5 \mathrm{~T}$ was applied and the upper and lower triangularity exhibited values of $\delta_{u}=0.11$ and $\delta_{l}=0.46$. In discharges \#28847 and \#28850, the MP-coils were operated at a constant current of $I_{\mathrm{MP}} \approx 1 \mathrm{kA}$ throughout the whole analyzed period (from $1.5 \mathrm{~s}$ to $7.6 \mathrm{~s}$ ).

The deuterium puff (figure 2(b)) was ramped in two steps with different slopes. This strong variation enabled us to achieve in all three discharges phases with type-I ELMs at the beginning, with small ELMs at higher densities - also without MPs due 
to the strong gas puff [16] — and a slow transition phase with both types of ELMs in between. The type-I ELMs were identified by the large spikes in the divertor current in figure $2(\mathrm{~d}-\mathrm{f})$, the amplitude of the small ELMs was strongly reduced. Complete type-I ELM suppression occurred earlier in the case with non-resonant than resonant MPs and latest without MPs. Yet, the line-averaged edge density (c) given by the peripheral channel of the DCN interferometer shows that the threshold for the occurrence of the last type-I ELM (marked by the horizontal lines) was almost the same for both discharges with MPs and close to the experimental criterion stated in [4], while it was slightly higher without MPs. In the fully mitigated regime, the density was the same in both perturbed discharges, but slightly lower compared to the reference.

\section{Kinetic edge profiles}

The effect of MPs on the electron edge kinetic data is visualized for some dedicated time points just before the occurrence of either a type-I or a small ELM crash in figure 3 and figure 4 which are each depicting the edge profiles of discharge \#28847 (left), \#28848 (middle) and \#28850 (right). From top to bottom the rows illustrate the electron temperature profiles (lines) together with the modelled ( $T_{\text {rad,mod, }}$ pluses) and the measured ( $T_{\text {rad,ECE }}$, crosses) radiation temperature values at the positions of their cold resonance, the corresponding residuals and the electron density and pressure profiles.

The good match of modelled and measured radiation temperatures and the small residual values indicated that the modelling worked well. The latter were defined by $\operatorname{res}_{\mathrm{i}, \mathrm{j}}=\left(T_{\mathrm{rad}, \mathrm{ECE}}(i, j)-T_{\text {rad,mod }}(i)\right) / \Delta T_{\text {rad,ECE }}(i, j)$ with channel $i$ and time point $j$ and their values mostly lying between \pm 1 stated that the modelled values matched the

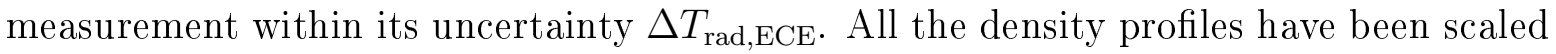
inwards within ECFM analysis (solid lines) as suggested by the separatrix criterion. In figure 3 the original density profiles are additionally plotted as dotted lines. The fact that the shift tended to be stronger by about $4 \mathrm{~mm}$ in the cases with MPs was consistent with the MP-coil setting (cf. section 3).

Figure 3 shows the profiles at an early time point $(t \approx 1.7 \mathrm{~s})$ which was dominated by type-I ELMs but exhibited also some small ELMs in between. To compare the profiles, the results of the reference discharge (middle) are again plotted as black dashed lines in the left and right column. This revealed almost identical profiles for the discharges without and with resonant MPs. To the contrary, the edge kinetic profiles of the discharge with non-resonant MPs differed significantly. They were strongly shifted inwards by about $10 \mathrm{~mm}$ and exhibited lower pedestal top values in temperature and density and a reduced edge temperature gradient resulting in a significantly flatter pressure gradient $(\approx-40 \%)$ compared to the reference discharge. This demonstrated that type-I ELMs were not related to a fixed edge pressure gradient but occurred at different values.

Additionally shown in green in figure 3 are the results of the two-line fit - a least- 

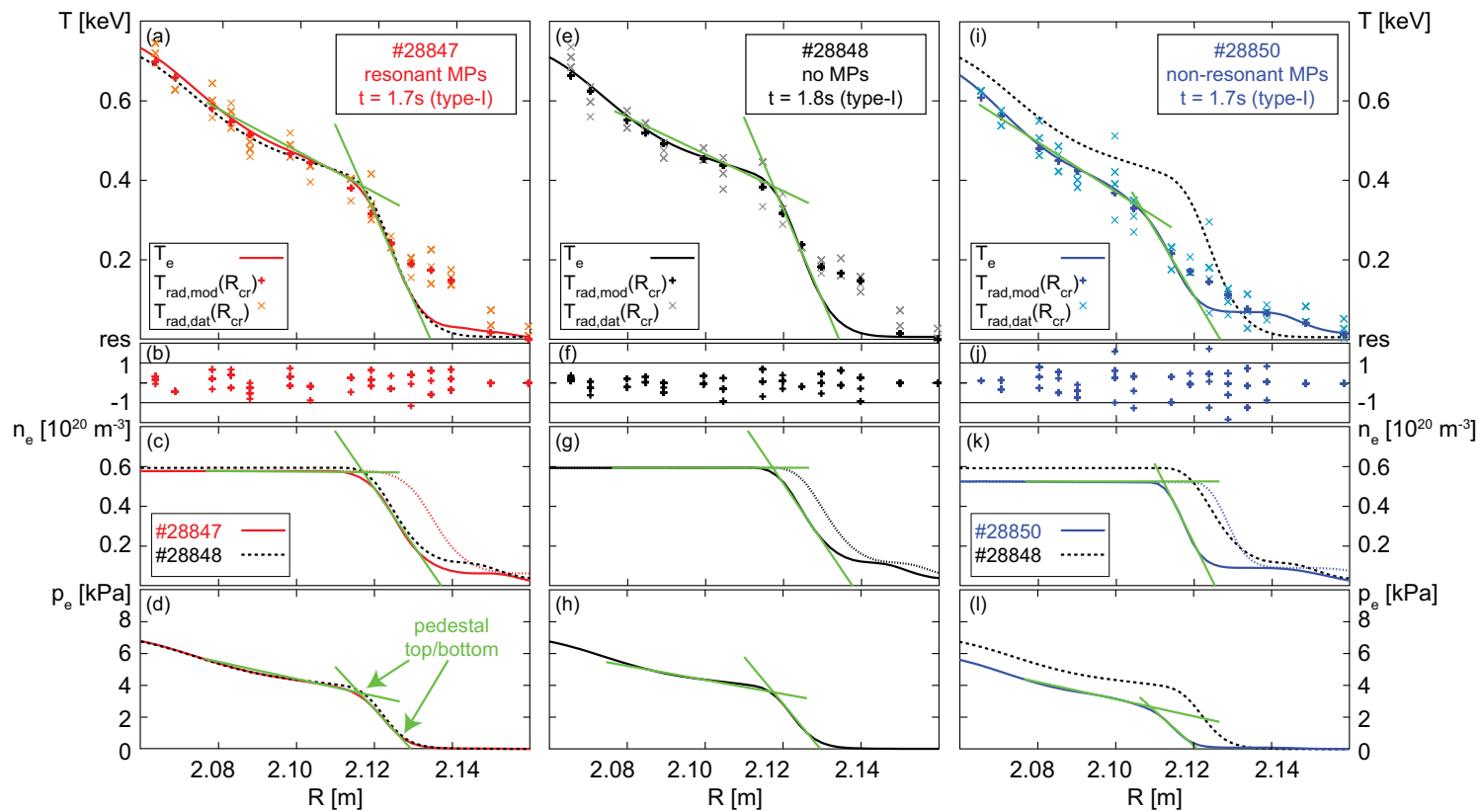

Figure 3. (a,e,i) Edge $T_{\mathrm{e}}$ profiles (lines) together with the modelled (pluses) and measured (crosses) $T_{\text {rad }}$ values at the positions of their cold resonance, $(\mathrm{b}, \mathrm{f}, \mathrm{j})$ their residuals, $(\mathrm{c}, \mathrm{g}, \mathrm{k})$ the applied (solid) and original (dotted) $n_{\mathrm{e}}$ and $(\mathrm{d}, \mathrm{h}, \mathrm{l}) p_{\mathrm{e}}$ profiles of discharges \#28847 (left), \#28848 (middle) and \#28850 (right) at $t \approx 1.7 \mathrm{~s}$; the black dashed lines in the left and the right column again show the profiles of discharge \#28848; the green lines additionally illustrate the two-line fits.
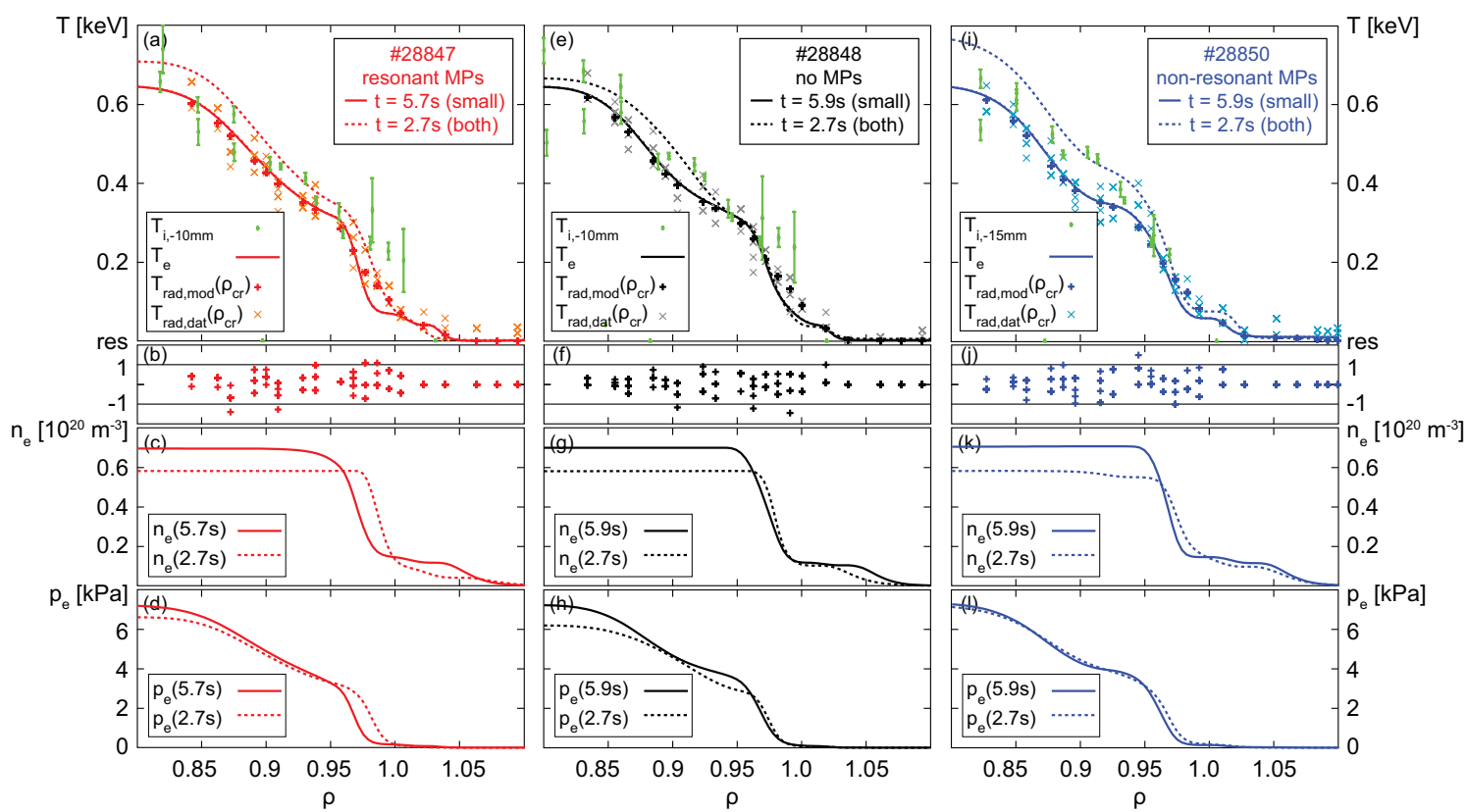

Figure 4. (a,e,i) Edge $T_{\mathrm{e}}$ (lines) and $T_{\mathrm{i}}$ (green dots) profiles together with the modelled (pluses) and measured (crosses) $T_{\text {rad }}$ values at the positions of their cold resonance, $(\mathrm{b}, \mathrm{f}, \mathrm{j})$ their residuals, $(\mathrm{c}, \mathrm{g}, \mathrm{k})$ the $n_{\mathrm{e}}$ and $(\mathrm{d}, \mathrm{h}, \mathrm{l}) p_{\mathrm{e}}$ profiles of discharges \#28847 (left), \#28848 (middle) and \#28850 (right) at $t \approx 2.7 \mathrm{~s}$ (dashed) and $t \approx 5.8 \mathrm{~s}$ (solid). 


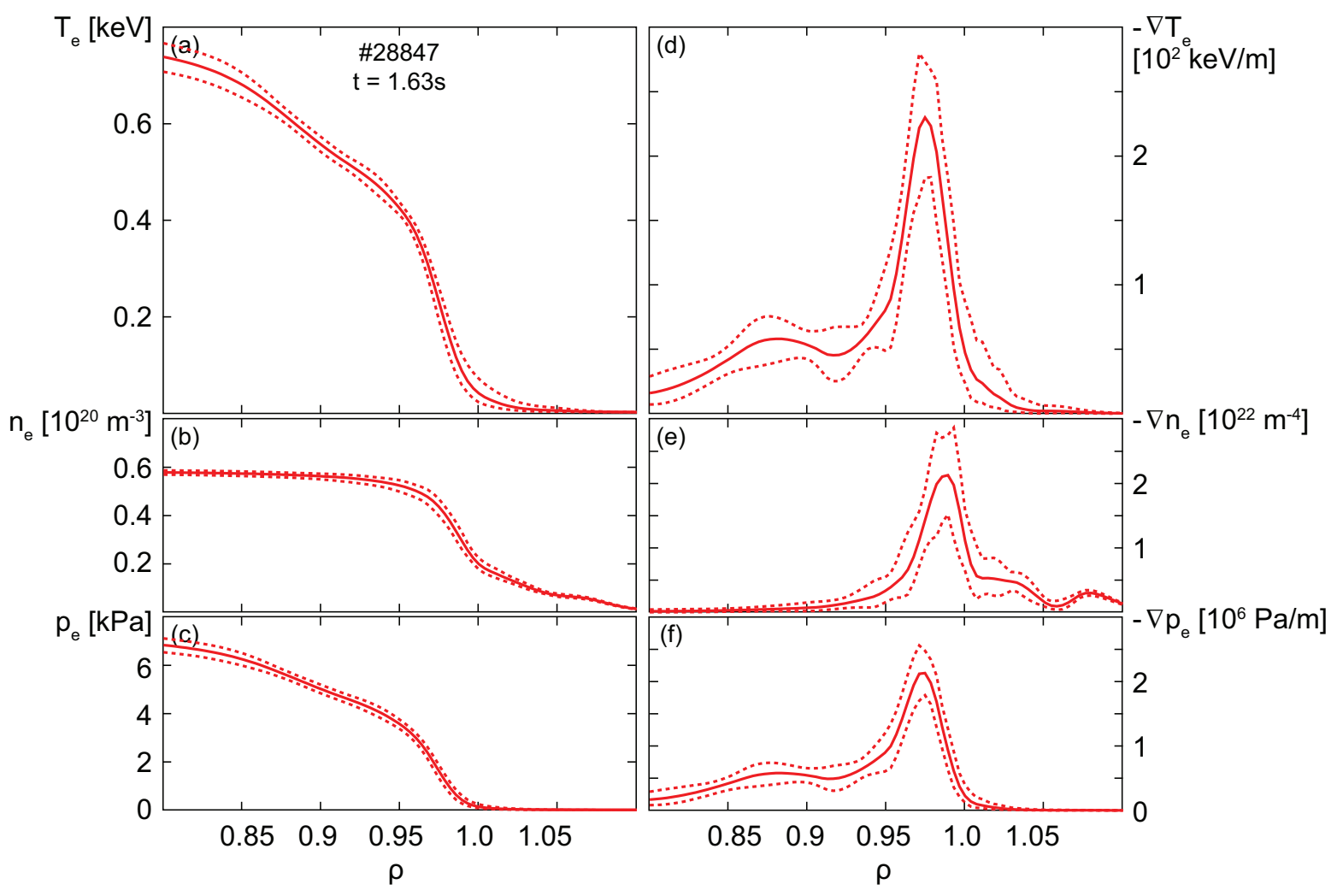

Figure 5. Edge profiles (solid) of (a) $T_{\mathrm{e}}$, (b) $n_{\mathrm{e}}$, (c) $p_{\mathrm{e}}$, (d) $\nabla T_{\mathrm{e}}$, (e) $\nabla n_{\mathrm{e}}$ and (f) $\nabla p_{\mathrm{e}}$ with their upper and lower boundaries (dashed) given by MCMC calculations of discharge $\# 28847$ at $t=1.67 \mathrm{~s}$.

squares fit that modelled the profiles between some centimetres inside the separatrix and the pedestal bottom - the point of maximum curvature - by two straight lines. This allowed for an automatic estimation of the pedestal top and bottom values and positions and the mean gradient between them. This information was used for the investigations presented in the next sections.

The profiles at a later time point $(t=2.7 \mathrm{~s})$ with strongly reduced type-I ELM frequency and small ELMs occurring in between the remaining type-I ELMs in all three discharges are shown as dashed lines in figure 4 - note here the use of the normalised poloidal radius $\rho=\sqrt{\left(\Psi-\Psi_{\text {axis }}\right) /\left(\Psi_{\text {separatrix }}-\Psi_{\text {axis }}\right)}$ with poloidal flux $\Psi$ as abscissae. These profiles are compared to the ones of a time point with fully suppressed type-I ELMs $(t \approx 5.8 \mathrm{~s})$ depicted as solid lines in figure 4 . At the later time point the pedestal top densities were higher in all three discharges due to the stronger gas puff. This caused a reduction of the temperature at and inside the pedestal top. Both these effects resulted in pressure profiles that were very similar to the previous ones. The comparison of the electron temperatures with the ion temperatures (green dots) from CXRS measurements at $t=2.7 \mathrm{~s}$ demonstrated that the profiles were - apart from an inward shift of the ion temperatures of about $\Delta R \approx 10 \mathrm{~mm}$ - almost the same. 
For the error analyses Markov Chain Monte Carlo (MCMC) calculations were performed. Shown exemplarily in figure 5 are the profiles (left, solid) and the gradients (right, solid) of the electron temperature (top), density (middle) and pressure (bottom) together with their upper and lower uncertainty bands (dashed). For the quantities that were used for further studies, the uncertainties exhibited values of $\Delta n_{\mathrm{e} \text {,pedtop }} \approx 5 \%$, $\Delta T_{\mathrm{e}, \text { pedtop }} \approx 5-10 \%$ and $\Delta \nabla p_{\mathrm{e} \text {,mean }} \approx 40 \%$.

To asses the uncertainty in the pressure gradient arising from the alignment between density and temperature profile, we performed a series of analyses with different shifts of the density profile relative to the temperature and investigated the variations of the maximal pressure gradient. Shifting the density by $\pm 10 \mathrm{~mm}$ around position which was suggested by the separatrix criterion - and which also shows a clear minimum in $\chi^{2}$ - only resulted in changes of the maximal pressure gradient of less than $5 \%$. This lies clearly within the uncertainty and therefore the alignment seems not to have a significant influence on the pressure gradient and the following results.

\section{Temporal evolution of the edge pressure gradient}

To study the temporal evolution of the edge pressure gradients in regimes with type-I and with small ELMs and compare it for the different settings of the MP coils, the two-line fit has been applied in several time frames of the three previous discharges. The mean edge pressure gradients are plotted in figure $6(\mathrm{a}, \mathrm{e}, \mathrm{i}, \mathrm{m})$ together with the divertor currents for four time intervals of each $50 \mathrm{~ms}$. The large gaps in the time traces of the pressure gradients originated from the lacking LIB data during chopped periods that were necessary for background signal subtraction. Smaller gaps also arose from time points at which the ECE data was not modelled correctly, for example due to an erroneous density profile or due to the bifurcation in modelling the radiation temperature in the SOL not as 'shine-through' radiation but as electron temperature (cf. [8]). These cases were automatically detected, e.g. by checking the residuals.

To generate a database for the pressure gradients, allowing a quantitative comparison of different discharges and time frames, the reduced values during ELMs and the following recovery phases had to be neglected and only the highest values some time before the next ELM had to be taken into account. These were at the same time the interesting ones for studying the stability threshold. However, an automatic detection of the small ELMs was - due to the small signal excursions - not possible. Therefore, the average of the upper $10 \%$-ile of the mean pressure gradient (named $\nabla p_{\text {e,upper }}$ ) within time intervals of $200 \mathrm{~ms}$ was used which is indicated by the horizontal lines in figure 6. Although this method produced a bias towards higher values leading to slightly overestimated pressure gradient threshold values, conclusions drawn from the comparison of different discharges or regimes, being exposed to the same uncertainty, were valid. In the following the behaviour of the edge pressure gradients in the four time frames is described: 

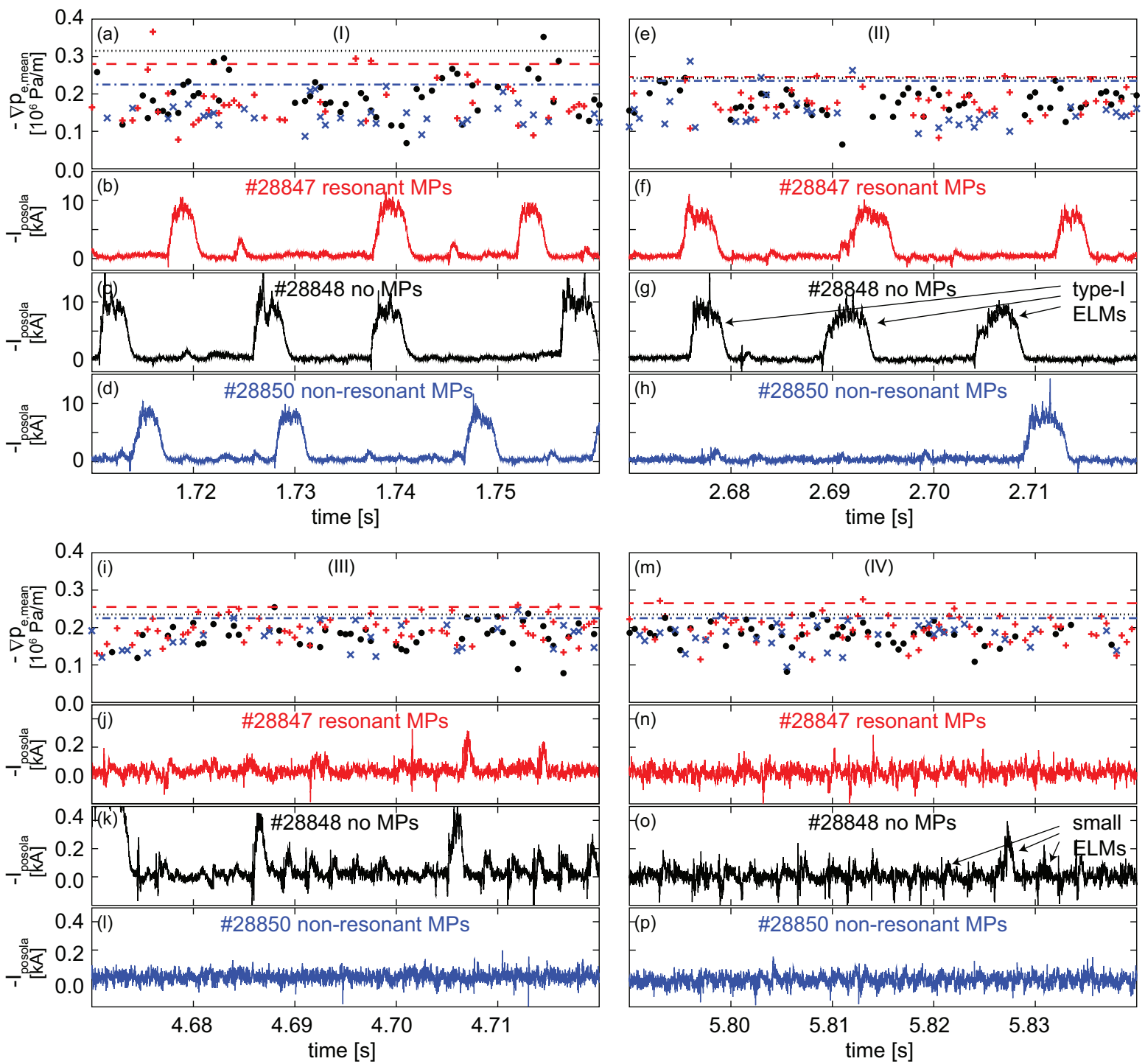

Figure 6. Time traces of the mean edge pressure gradients $(\mathrm{a}, \mathrm{e}, \mathrm{i}, \mathrm{m})$ of discharges \#28847 (red pluses), \#28848 (black circles) and \#28850 (blue crosses) together with the corresponding divertor currents; the horizontal lines indicate the mean of the $10 \%$ highest pressure gradient values.

Time slot (I). All discharges were dominated by type-I ELMs but also some small ELMs occurred and the discharges with MPs already showed a reduced type-I ELM frequency. The discharges without and with resonant MPs showed a very similar behaviour with significantly higher values of the pressure gradient before type-I than before small ELMs and with only a slight difference $(\approx 10 \%)$ in the upper value. Yet, the pressure gradients of the discharge with non-resonant MPs were strongly reduced and did not reveal a difference in the pre-type-I and pre-small ELM values. The upper value of the pressure gradient was significantly reduced $(\approx-40 \%)$ compared to the reference discharge. 
Time slot (II). The discharges without and with resonant MPs were dominated by type-I ELMs, while the discharge with non-resonant MPs was dominated by small ELMs. The upper values of the pressure gradient in the discharges without and with resonant MPs were - compared to time slot (I) — reduced and reached similar values for all three discharges.

Time slot (III). The MP discharges exhibited small ELMs only, while the reference discharge was dominated by small ELMs, but exhibited some type-I ELMs as well. Also the small ELMs showed larger excursion in the divertor current compared to the discharges with MPs. Again, the upper values of the pressure gradient were similar at the low level of time slot (II) - for all three discharges.

Time slot $(I V)$. All three discharges exhibited small ELMs only. Again the excursions in the divertor signal were the largest for the reference discharge and the smallest for the discharge with non-resonant MPs. The upper values of the pressure gradient were similar for these two discharges and tended to be slightly higher for the discharge with resonant MPs.

\section{Relation of local edge parameters with ELM type}

The previous findings are summarized in figure 7 in order to demonstrate how the occurring ELM type and the edge kinetic parameters depend on the external settings of MP-coil configuration, deuterium puff and heating power. The type-I ELM frequencies (a), the pedestal top densities (b) and temperatures (c) and the upper values of the mean pressure gradients $(d)$ are plotted against the amount of gas puff. The data shows the mean values of different time slots of $200 \mathrm{~ms}$ each with type-I ELMs only (filled squares), both types of ELMs (empty squares) and small ELMs only (crosses) of the previous triplet of discharges together with the data of a similar discharge without MPs and higher heating power (\#28863) shown in brown.

Type-I ELM frequency versus deuterium puff (a). In all discharges the frequency of the type-I ELMs decreased with increasing gas puff until they were suppressed completely. The decay was faster with lower heating power (compare black and brown shaded lines). For the same heating power, the type-I ELM frequency was generally lower with than without MPs (compare black and red shaded lines).

Pedestal top density versus deuterium puff (b). The density strongly rose with increasing gas puff but in the discharges with low heating power (black shaded line) it saturated at high gas puff. Data points above the vertical dashed line were affected by the ECE cut-off and omitted in the following plots.

Pedestal top temperature versus deuterium puff (c). Due to its cooling effect, stronger gas puff - at otherwise same conditions - resulted in lower pedestal top temperatures (cf. black shaded line). Higher heating power at the same gas puff caused higher temperatures (cf. brown points). At a sufficiently high level of gas puff, discharges 

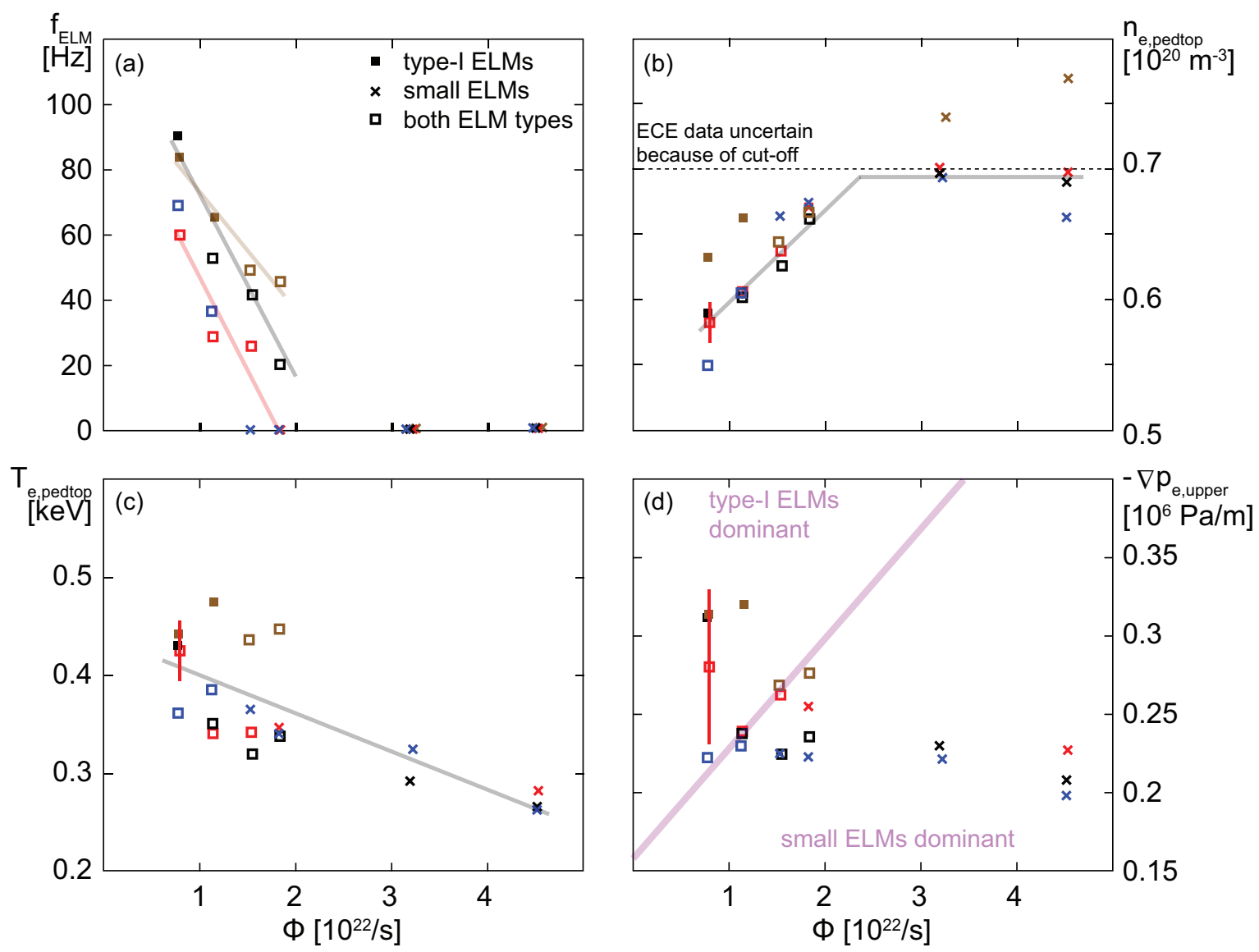

Figure 7. (a) Type-I ELM frequency, (b) pedestal top density, (c) pedestal top temperature and (d) upper envelop of the edge pressure gradient versus deuterium puff for discharges \#28847 (red), \#28848 (black), \#28850 (blue) and \#28863 (brown); different symbols indicate the ELM type; additionally plotted are lines to guide the eye.

with identical plasma settings generally exhibited similar pedestal top temperatures with and without MPs.

Upper envelop of the pressure gradient versus deuterium puff (d). The evolution of density and temperature either compensated each other such that the pressure gradient was almost constant or it was slightly reduced with stronger gas puff. Generally, at a sufficiently high level of gas puff, there was no significant difference between discharges with and without MPs. It can clearly be seen that in regimes with low gas puff and high pressure gradient (above purple line), type-I ELMs were the dominant ones.

Operationally, application of MPs or a large gas puff can result in mitigation of ELMs. However, the identification of the actual (physical) reason for ELM mitigation requires the detection of dependencies between internal plasma parameters and the ELM type. To study whether large and small ELMs populate separate edge parameter regions, independently of which actuator is used to access them, the upper envelop values of the pressure gradients of several discharges with characteristics listed in table 1 are plotted versus the pedestal top densities (a) and temperatures (b) in figure 8. The different 


\begin{tabular}{c|c|c|c} 
Discharge & MP-coil setting & $P_{\text {NBI }}[\mathrm{MW}]$ & Color \\
\hline$\# 27941$ & $n=1$ resonant & 9.7 & orange \\
$\# 27942$ & - & 9.4 & grey \\
$\# 27943$ & $n=1$ non-resonant & 8.7 & light blue \\
$\# 28847$ & $n=2$ resonant & 4.9 & red \\
$\# 28848$ & - & 4.9 & black \\
$\# 28850$ & $n=2$ non-resonant & 4.9 & blue \\
$\# 28863$ & - & 7.4 & brown \\
$\# 29231 / 32 / 33 / 38$ & $n=2$ resonant & $4.7-12.1$ & dark red \\
$\# 29231 / 32 / 33 / 38$ & - & $4.7-12.1$ & dark grey
\end{tabular}

Table 1. Characteristics of discharges shown in figure 8
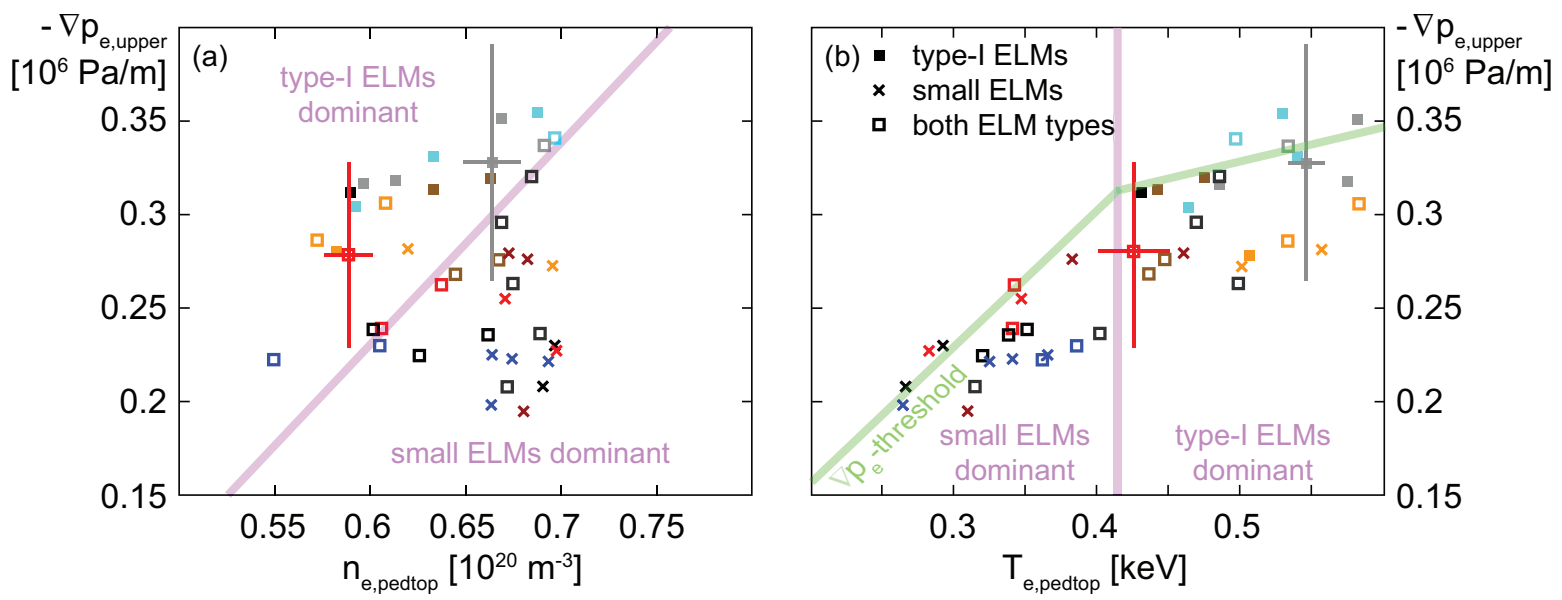

Figure 8. Upper envelop of the edge pressure gradient versus (a) the density and (b) the temperature at the pedestal top for various discharges shown in different colours; different symbols indicate the ELM type; additionally plotted are lines to guide the eye.

ELM types are marked by different symbols. For two data points (red and grey), the uncertainties obtained from MCMC methods are indicated.

Upper envelop of the pressure gradient versus pedestal top density (a). This plot can be divided in two regions according to the occurrence of ELM type as indicated by the purple shaded line. Type-I ELMs clearly dominated at higher pressure gradients and lower pedestal top densities, while at lower pressure gradients and higher densities, mostly small ELMs occurred. This behaviour was independent of the external settings, however application of MPs provoked small ELMs also at rather low densities (red and orange) and non-MP cases also showed type-I ELMs at rather high densities and low pressure gradients (black and dark grey). The indicated uncertainties demonstrated that although they are still too large to resolve small variations - especially in the pressure gradient - , the differences between the two regions were significant. 
Upper envelop of the pressure gradient versus pedestal top temperature (b). There seemed to exist an upper threshold for the pressure gradient (green shaded line) in both ELM regimes which increased with the pedestal top temperature. The small ELMs mainly occurred at a colder pedestal top (left of the purple shaded line), the region of higher pedestal top temperatures and pressure gradients was dominated by type-I ELMs. Application of MPs resulted in a higher threshold temperature between the type-I and small ELM regimes.

\section{Discussion}

Experiments have been performed to study the transition from type-I to small ELMs by increasing the gas fuelling rate or applying MPs - and the associated edge kinetic data. Small ELMs generally occur at higher pedestal top densities, reduced pedestal top temperatures and flattened edge pressure gradients compared to regimes with dominant type-I ELMs (cf. figure 8). These results are now discussed in the light of two hypotheses been invoked to explain ELM mitigation via MPs.

Hypothesis "Edge cooling": Peeling-ballooning stabilization due to edge cooling. It has been shown that ELMs can be mitigated not only by the application of MPs but also via edge cooling with neutral gas. The latter is ionized in the Scrape-Off Layer (SOL) which reduces the local temperature. In the presence of high densities, hence small temperature gradients, this also decreases the pedestal top temperature which can lead to the transition from type-I to type-III ELMs [16]. MPs might act in a similar way by providing an additional source of cold particles.

The screening of the perturbation fields in the SOL is much less effective than in the confined plasma due to the higher resistivity of the cold SOL plasma and the target plates. Hence, helical distortion of the SOL magnetic field is very likely. In fact, observations of a split power deposition pattern on the target plates ('strike-line splitting') are consistent with vacuum calculations [17]. Furthermore, application of MPs causes a finger-like structure in the edge field lines reaching the SOL and connecting confined and non-confined regions. Additionally with the observation of a high density region at the high field side [18] it is conceivable that ELM mitigation via MPs is achieved by the creation of field line structures that overlap with the high density region which increases the particle flux from the cold SOL to the edge plasma [19].

In fact, small ELMs generally occur — with and without MPs — in regimes with higher pedestal top densities and lower pedestal top temperatures compared to type-I ELMy regimes (cf. figure 8). However, the effect of MPs seems not to be the provision of an additional source of cold particles from the SOL region. This is indicated by figure 7(b) which shows that the densities with applied MPs are not generally higher than without. Hence, reaching a certain density value might not be sufficient - though necessary - for ELM mitigation. This is also reflected in figure 8(a) which shows fully mitigated ELMs only for $n_{\text {e,pedtop }} \gtrsim 6.6 \times 10^{19} \mathrm{~m}^{-3}$, while also type-I ELMs occur in this 


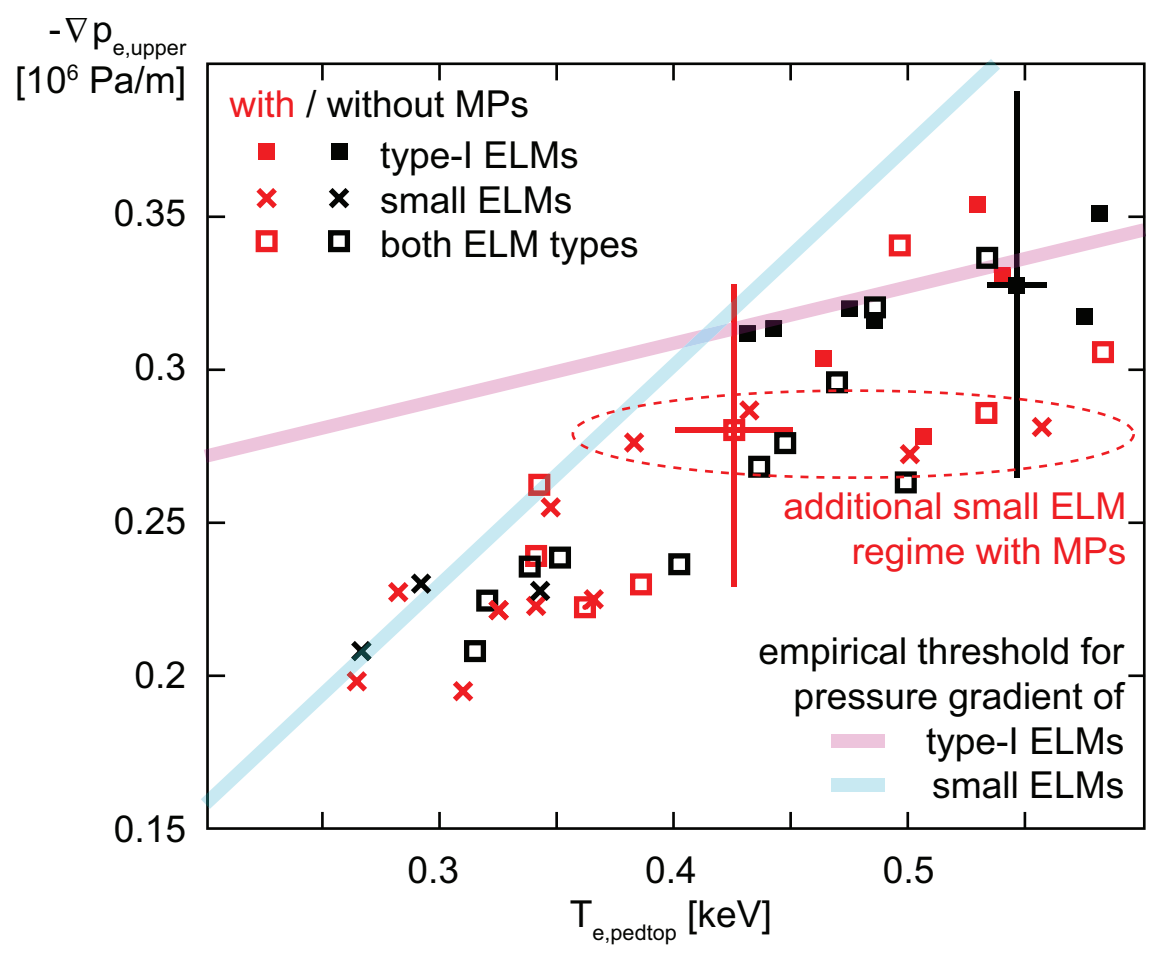

Figure 9. Upper envelop of the edge pressure gradient versus pedestal top temperature for various discharges with (red) and without (black) MPs; the different symbols indicate the ELM type; additionally plotted are lines to guide the eye.

density regime.

Furthermore, also the temperature is not generally lower in the presence of MPs (cf. figure 7(c)). Finally, with MPs there exists a regime with small ELMs also at higher temperatures (cf. figure 8(b)), demonstrating that application of MPs allows to access mitigated ELM regimes without significant edge cooling.

Hypothesis "Stability boundary shift": Shifted stability threshold of small ELMs below type-I ELM threshold. Another possibility is that MPs are not affecting the plasma parameters directly but acting on the stability limit of type-I ELMs and/or small ELMs. The decision for the occurring ELM type depends on which stability limit is reached first - i.e. which type of ELM exhibits the lower stability threshold. The presence of that ELM type, prevents the other by clamping the edge profiles to the lower stability limit. In the presence of MPs, the stability boundary of either ELM type might be shifted such that small ELMs occur in regimes that are usually — without MPs — dominated by type-I ELMs [20, 14].

In figure 9 the same data as in figure 8 is shown but for easier comparison the colourcoding here distinguishes only cases with (red) and without MPs (black). To guide the eye, two lines indicating empirical edge pressure gradients thresholds for type-I ELMs (purple) and small ELMs (blue) are sketched.

According to this image, in regions of colder edge plasma the threshold for small 
ELMs is lower than the type-I ELM threshold, i.e. small ELMs are triggered before the limit for type-I ELMs is reached and the latter are suppressed - vice versa at high edge temperatures. The closer both thresholds are, the higher is the probability for coexisting type-I and small ELMs. The dependency of the occurring ELM type on the temperature might be a sign for the small ELMs being a resistive instability since the resistivity increases with decreasing temperature $\left(\eta \propto T^{-1.5}\right)[21]$.

A possible effect of the MPs might be a shift of the small ELM stability limit towards lower pressure gradients at higher edge temperatures. This is indicated by the occurrence of a regime with small ELMs at temperatures in the range of $T_{\mathrm{e} \text {,pedtop }}=350-550 \mathrm{eV}$ when MPs are applied, while without MPs complete type-I ELM suppression is only observed below $T_{\text {e,pedtop }}=350 \mathrm{eV}$.

Another mechanism that has been invoked to explain the DIII-D ELM suppression experiments in their early days [3] assumes that enhanced radial transport due to ergodization may flatten the edge pedestal pressure profile to the point where the stability limit for type-I ELMs is no longer reached and the latter are suppressed. First experiments at ASDEX Upgrade indicate that the degree of ergodization is not sufficient to open up an additional channel of radial transport which keeps the pressure below the ballooning limit. Further studies on this topic are ongoing.

Summarizing all these findings, the following picture about the dependencies of the edge parameters and the occurrence of ELM type emerges: Heating power and gas puff determine density and temperature. These are thereby coupled via the pressure gradient that is clamped by the ELM stability limit. The ELM type and its corresponding pressure gradient threshold in turn depend on the pedestal temperature.

Up to now, only the differences between the small ELMs and type-I ELMs have been discussed. However, it would also be interesting to know whether the small ELMs might be identified with or clearly distinguished from another ELM type, especially type-II and type-III ELMs. The present work allows the comparison of small ELMs in the presence of MPs (from now on: MP-mitigated ELMs) with the ones only caused by a strong gas puff (without MPs) — which are supposed to be type-III ELMs [16]. In fact, there are many similarities between the MP-mitigated ELMs and the type-III ELMs. Both of them only exist at a sufficiently high gas puff relative to the heating power. Furthermore, they are both related to regimes with higher edge density and reduced edge temperature and pressure gradient. Although the 'threshold' values of these external and local edge parameters are not the same for the occurrence of MP-mitigated ELMs and type-III ELMs, the transition from type-I to both small ELM regimes follows the same trend in the mentioned parameters. This might be an indication that MP-mitigated ELMs and type-III ELMs are caused by the same instability with a slightly different stability threshold in the presence of MPs. Nevertheless, type-III ELMs exhibit two typical features that have not been observed for the MP-mitigated ELMs [22]: A magnetic precursor and the decreasing poloidal velocity of the modes related to the occurrence of each ELM event. However, in [22] also significant differences between MP-mitigated 
ELMs and type-II ELMs have been found. The first do neither show the continuous fluctuations nor the poloidal asymmetry in the mode amplitude of the latter.

In conclusion, we demonstrated to possess a valuable tool to reveal the small changes in the edge kinetic data in different ELM regimes with and without MPs. These indicate that in the high collisionality regime at ASDEX Upgrade the effect of MPs rather consists in a change in the stability limit than in an enhanced heat transport or an additional particle source.

\section{References}

[1] Hender T C et al 1992 Nucl. Fusion 32 (12) 2091

[2] Miura Y et al 1991 Proc. 13th International Conference on Plasma Physics and Controlled Nuclear Fusion Research (Washington, USA) 1 (IAEA-CN-53) 325

[3] Evans T E et al 2004 Phys. Rev. Lett. 92 (23) 235003

[4] Suttrop W et al 2011 Plasma Phys. Control. Fusion 53 (12) 124014

[5] Fischer R, Fuchs C J, Kurzan B, Suttrop W, Wolfrum E and the ASDEX Upgrade Team 2010 Fusion Science and Technology $\mathbf{5 8} 675$

[6] Rathgeber S K, Barrera L, Eich T, Fischer R, Nold B, Suttrop W, Willensdorfer M, Wolfrum E and the ASDEX Upgrade Team 2013 Plasma Phys. Control. Fusion 55 (2) 025004

[7] Rathgeber S K, Barrera L, Birkenmeier G, Fischer R, Suttrop W and the ASDEX Upgrade Team 2013 Proc. 40th EPS Conference on Plasma Physics (Espoo, Finland) 37D P4.108

[8] Rathgeber S K 2013 Electron temperature and pressure at the edge of ASDEX Upgrade plasmas $\mathrm{PhD}$ Thesis Ludwig-Maximilians-Universität München

[9] Fischer R, Wolfrum E, Schweinzer J and the ASDEX Upgrade Team 2008 Plasma Phys. Control. Fusion $\mathbf{5 0} 085009$

[10] Luhmann N C Jr et al 2008 Fusion Sci. Technol. 53335

[11] Neuhauser J et al 2002 Plasma Phys. Control. Fusion 44855

[12] Schneider P A et al 2012 Plasma Phys. Control. Fusion 54105009

[13] Suttrop W et al 2013 Fus. Eng. Des. 88 (6-8) 446

[14] Suttrop W et al 2012 Proc. 24th Int. Conf. on Fusion Energy (San Diego, 2012) [EX/3-4]

[15] Fischer R, Fuchs C J, McDermott R, Rathgeber S K, Suttrop W, Willensdorfer M, Wolfrum E and the ASDEX Upgrade Team 2012 Plasma Phys. Control. Fusion 54 (11) 115008

[16] Suttrop W et al 1997 Plasma Phys. Control. Fusion 39 (12) 2051

[17] Lunt T et al 2012 Nucl. Fusion 52 (5) 054013

[18] Potzel S, Wischmeier M, Bernert M, Dux R, Müller H W, Scarabosio A and the ASDEX Upgrade Team 2013 J. Nucl. Mater. 438285

[19] Wolfrum E, Lunt T, Müller H W, Potzel S, Wischmeier M, Suttrop W and the ASDEX Upgrade Team 2013 Proc. 40th EPS Conference on Plasma Physics (Espoo, Finland) 37D P4.105

[20] Evans T E et al 2005 Nucl. Fusion 45595

[21] Ferraro N M, Jardin S C and Snyder P B 2010 Physics of Plasmas 17 (10) 102508

[22] Boom J 2012 Characterization of edge localized modes in tokamak plasmas PhD Thesis Technische Universiteit Eindhoven 